\title{
LDL and beyond: New emerging LDL biomarkers in lipidology
}

\author{
Dr. Sikandar Hayat Khan* \\ Department of Pathology, PNS HAFEEZ, Islamabad, Pakistan \\ ${ }^{*}$ Corresponding author: Dr. Sikandar Hayat Khan, Department of Pathology, PNS HAFEEZ, Islamabad, Pakistan; Email: sik_cpsp@yahoo.com
}

Received: April 12, 2019; Accepted: April 22, 2019; Published: May 14, 2019;

\begin{abstract}
Lipidology as super-specialty is evolving both in terms of risk prediction but also to uncover the hidden mysteries within humans suffering from atherosclerotic cardiovascular disease (ASCVD) associated complication with apparently similar LDL concentration and particle size. Over decades since LDL discovery in 1950, the science has covered miles to allow us to learn more about the villainous nature of LDL lipoprotein i.e., ApoB, size wise fractions of LDL particles especially the small dense and large buoyant LDL types and oxidized LDLs. However, the recent evidence suggest exploring the morphology of LDLp within plaques suggest the varying concentration of sphingolipids to phosphatidylcholine in LDL-aggregates. This discovery has allowed newer insights into the pathophysiological mechanisms leading to plaque instability and rupture though an accelerated atherosclerotic mechanistic phenomena. This newer development will also allow us to segregate individuals with similar LDL phenotypes in terms of concentration and particle size to end up with ASCVD related complications. This brief communication discusses briefly discusses the recent LDL-plaque relationship and highlights new lipid biomarkers to further allow personalized segregation of cardiovascular disease (CVD) risk.
\end{abstract}

Key words: LDL-cholesterol (LDLc), small dense LDL-cholesterol (sdLDLc), Large buoyant LDL-cholesterol (lbLDLc), LDL-aggregates, Oxidized LDL, Lipoprotein associated phospholipase A2 (Lp-LPA2), ApoB

\section{Introduction}

While cholesterol was acknowledged as one of the components being present in the blood from $16^{\text {th }}$ century onwards, it was Oncley et al in 1950 who isolated the beta globulin from fraction-III by means of ultracentrifugation. [1] Since then it was realized that the increasing LDL lipoprotein concentration emerged strongly as a risk for various atherosclerotic cardiovascular diseases (ASCVD) and was thus included as a primary prevention target parameter. [2] Though multiple studies have highlighted LDL lipoprotein concentration as the culprit, but later research further dissected LDL fractions to identify particle size to be more related with ASCVD. [3] Down the line researchers were able to segregate LDL particles between two broad categories including small dense LDL particles (sdLDc) and large buoyant LDL particles (lbLDLc), where the former category is associated with more atherogenicity and ASCVD. [4] Guidelines followed the initial research and quickly adopted the concept of particle size and some labs even marketed the LDL-particle size as of now. [5] The traditional concept of LDL cholesterol concentration measurements is still, however in vogue across the world and evolved from calculation based methods to directly measuring techniques which have improved at least the precision of LDL measurement. [6] Form the point of view developing and under developed economies the strategy still remains the most cost-effective, well-understood in terms of data interpretation and feasibility in terms of instrument availability. While the reliance on conventional lipid profile data currently seem to be the logical option for many set ups across the globe still, there are gaps with this "LDL concentration approach" to predict ASCVD risk. [7] LDL Lipoprotein structure has more to offer, than just the cholesterol content as the origin from VLDL to movement within circulation and with dumping down physiologically through LDL receptors into liver and pathologically into vasculature is highly variable between subjects. [8] Data suggest simple LDL concentration measures does not provide optimal appraisal of ASCVD in many subjects. Ramasamy et al in his very recent publication has clearly highlighted the limitations in lipid measurement technologies to highlight the need to develop biomarkers to better predict cardio vascular disease (CVD) risk. [7] Lawler et al using Nuclear Magnetic Resonance (NMR) Spectroscopy evaluated different fractions of LDL particles and concluded that small LDL particle was associated with CVD risk.[9] Finally literature at least now clearly acknowledges the LDL sub-fractions to be differently linked with ASCVD, and the whole lipoprotein risk evaluation using traditional lipid markers are poorly equated with future CVD prediction. [10]

\section{Emerging biomarkers in Lipidology}

\section{a. Small dense LDL-cholesterol (sdLDLc)}

The initial search comes in through discovery of LDLfractions where an initial broader categorization was made as to segregate LDL particles into two categories i.e., sdLDLc and large buoyant LDL cholesterol (lbLDLc). sdLDLc in current research has been considered as risk for CVD. [11] However, lbLDLc were not considered atherogenic which clearly 
challenges the use of LDLc in clinics for identifying ASCVD risk.

\section{b. ApoB measurements}

Alongside the protein components within lipoprotein also entered clinical market as ApoA as surrogate for HDLc and ApoB for LDLc. The Insulin Resistance Atherosclerosis Study (IRAS) have graded ApoB measurements to be more predicative than LDLc.[12] However, research shows ApoB not to provide any additional information than conventional LDLc. $[13,14]$

\section{c. Lipoprotein associated phospholipase A2 (Lp-LPA2)}

This enzyme is found mainly in LDLc where it helps contributes to atherosclerosis but confers some anti-atherogenic advantages to HDLc as well. Lp-PLA(2) studies collaboration group have identified a strong association of enzyme activity and mass with various ASCVD adverse outcomes like stroke, heart diseases and hypertension. Similarly, Anderson J et al have demonstrated Lp-LPA2 as an independent risk factor for predicting coronary artery disease (CAD). [16] Though appealing in terms of its role to cleave oxidized phospholipids and acting as a chemo-attractant to bring inflammatory proteins and cells to unstable plaque, still large trials like JUPITER and HPS have not found additional benefit of its utilization for both primary and secondary prevention of ASCVD than conventional LDLc. [17,18] Another issues haunting Lp-PLA(2) is the measurement variability due to assay formats, which stands mandatory before its clinical use in routine. [19] So it seems that Lp-PLA(2) use in clinical arena is bound to face delays or may never be used due to incoming better markers.

\section{d. LDL Particles}

Over the last 2 decades LDL particles have been found to have multiple sizes, where the literature has identified varying atherogenic potential for LDL-sub particles. Gourgari et al have identified in a study LDL-particle size to be higher in polycystic ovarian syndrome subjects (PCOS) in comparison to controls which was related with markers of inflammation and insulin resistance. [20] Similarly others have highlighted LDL particles to be more related with ASCVD. [3] However, the contrasting evidence highlighted in the Multi-Ethnic Study of Atherosclerosis(MESA) observed slightly greater benefit by using LDLp/HDLp ratio but identified this risk prediction for coronary heart disease (CHD) to get attenuated after adjustment of standard lipid variables. [21]

\section{e. Oxidized LDL}

For some time researchers did thrive on the concept of LDL concentration and particle size, but emerging evidence from kinetic studies identified various post-translational modifications like oxidative changes. [22, 23] These oxidized LDL (oxLDLc) are considered to result in certain "damage associated molecular patterns" (DAMP), which are later to result in vascular inflammation. [22] So oxLDLc within vessel walls can act as new LDL biomarkers; however, no standardized lipid lowering therapy is yet available to prevent this oxidative damage in LDL.[23]

\section{f. LDL-aggregates}

Within vessel wall it has been demonstrated that LDL particles aggregate. [24] These aggregates of LDL particles within arterial walls are quite atherogenic and can cause changes like conversion of macrophages into foam cells and accumulation within smooth muscles to cause accelerated atherosclerosis and plaque formation by the enzyme sphingomyelinase (SMase). [24, 23] LDL-aggregates, though not in correlation with conventional lipid and inflammatory markers but still have been observed to change with lifestyle modifications, use of PCSK9 inhibitors and other treatment modalities. These LDL-aggregates are distinguished by the fact that they have increase sphingolipids to phospatidylcholine ratio, which accelerates the process of atherosclerosis and in turn predispose plaques to rupture.Therefore, LDL-aggregates may emerge as powerful diagnostic and monitoring tool in future. [23-25]

\section{Futuristic incorporation in lipid clinic care path- ways}

While current clinical market poses both economic issues and lack of quality research, still visibility is now here that conventional lipid markers are not able to predict ASCVD in multiple cases and the need is ever appreciated for advance lipid biomarkers to address both personalized medicine and health economics. The below mentioned algorithm is meant for a dedicated lipid clinic where an individualized diagnosis of lipid pathology could be diagnosed to avoid pan-medical trials and to provide specific interventional approached to reduce ASCVD risk for the patients and genetic solutions for the family members.

This data, albeit discussed recently in literature replies to the critical question raised in the clinics that "why ASCVD prevalence did not correspond with LDL concentration and particle size?” Deeper insight intoLDLp interaction within plaque, ratio of sphingolipid / phosphatidylcholine as prevails within LDLp and the activity of sphingomyelinase (SMase) all finally converge towards plaque progression, rupture and thus the acute consequences resulting from the ASCVD. It is anticipated that SMase activity and genetic alterations in LDL aggregation will probably follow these phenotypic changes to clarify the mutations and polymorphisms underlying the varying development of plaques and onward ASCVD risk among individuals.

\section{Closing remarks}

Incorporation overtime to address one of the crucial villains to cause ASCVD would require additional biomarker arsenal to allow meaningful data to segregate risk prediction among individuals with similarities baseline LDL phenotypes i.e., Aggregation-prone LDLp and Aggregation-resistant LDLp. In this regard advanced lipid clinics can extend help to incorporate LDL particle measurements, 
phenotyping of LDL classes, functional assays to asses to learn LDL aggregation and oxidized LDL types. Molecular diagnostics can also be added to specifically diagnose the underlying genetic pathology. A one-time assessment can help predict risk for ASCVD related morbidity and mortality along with avoiding people with unnecessary lifelong medication, concerns and as a very powerful primary prevention tool. Perhaps larger tertiary care set ups in country should develop tools and arsenals to perform advanced lipid testing within dedicated lipid clinics to address the multifactorial pathogenesis of
ASCVD to address the pushing needs to "personalized medicine", cost-effective care provision and finally to segregate patients who need lipid lowering treatment or otherwise.

Consent for publication: Not applicable (No individual data was presented)

Competing interests: The author has no competing interests to declare.

Data funding: There are no funding sources to disclose.

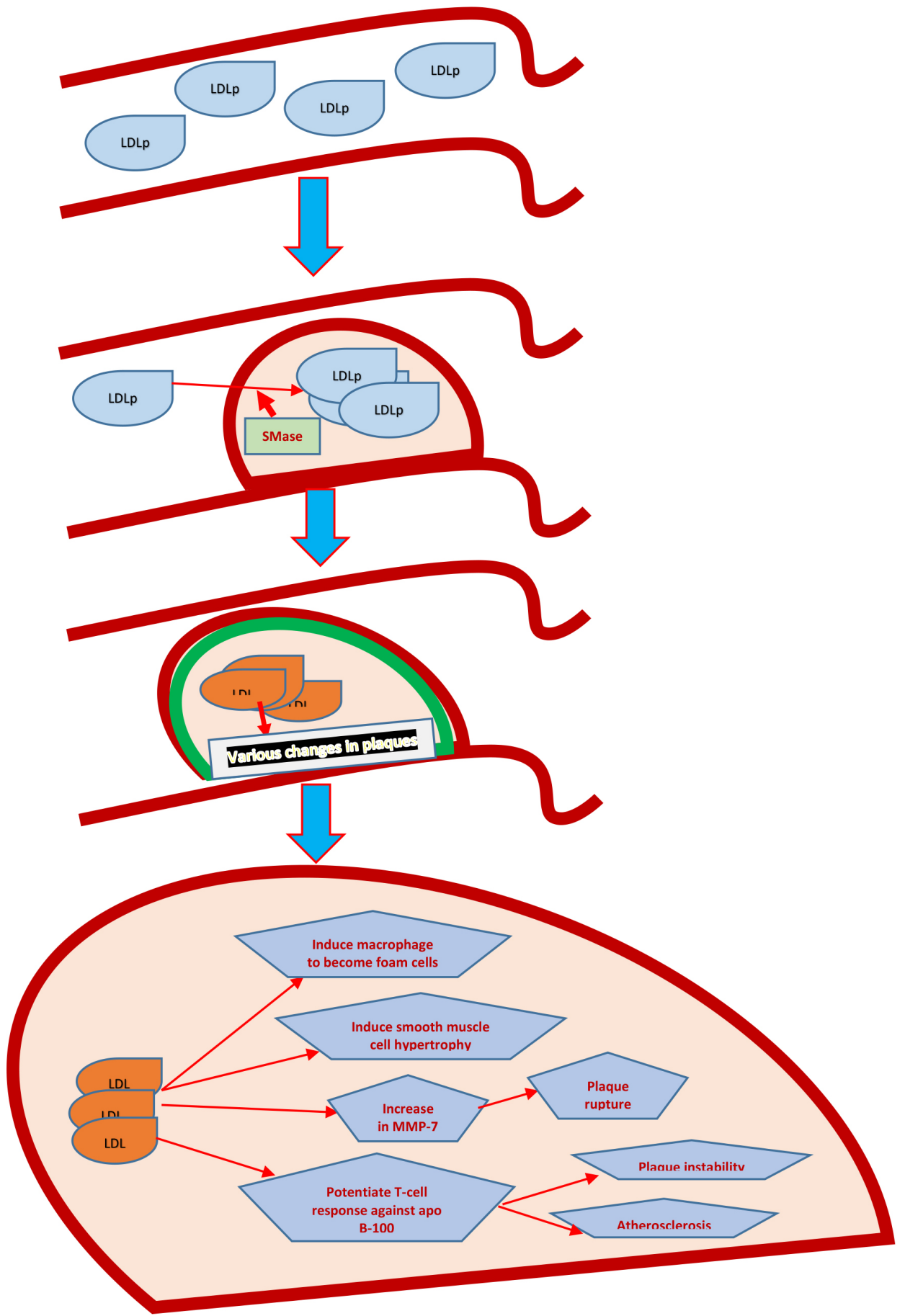

Figure 1. The process of LDLp entry into carotid intima, to changeswithin the plaque resulting in plaque instability and onward rupture. 


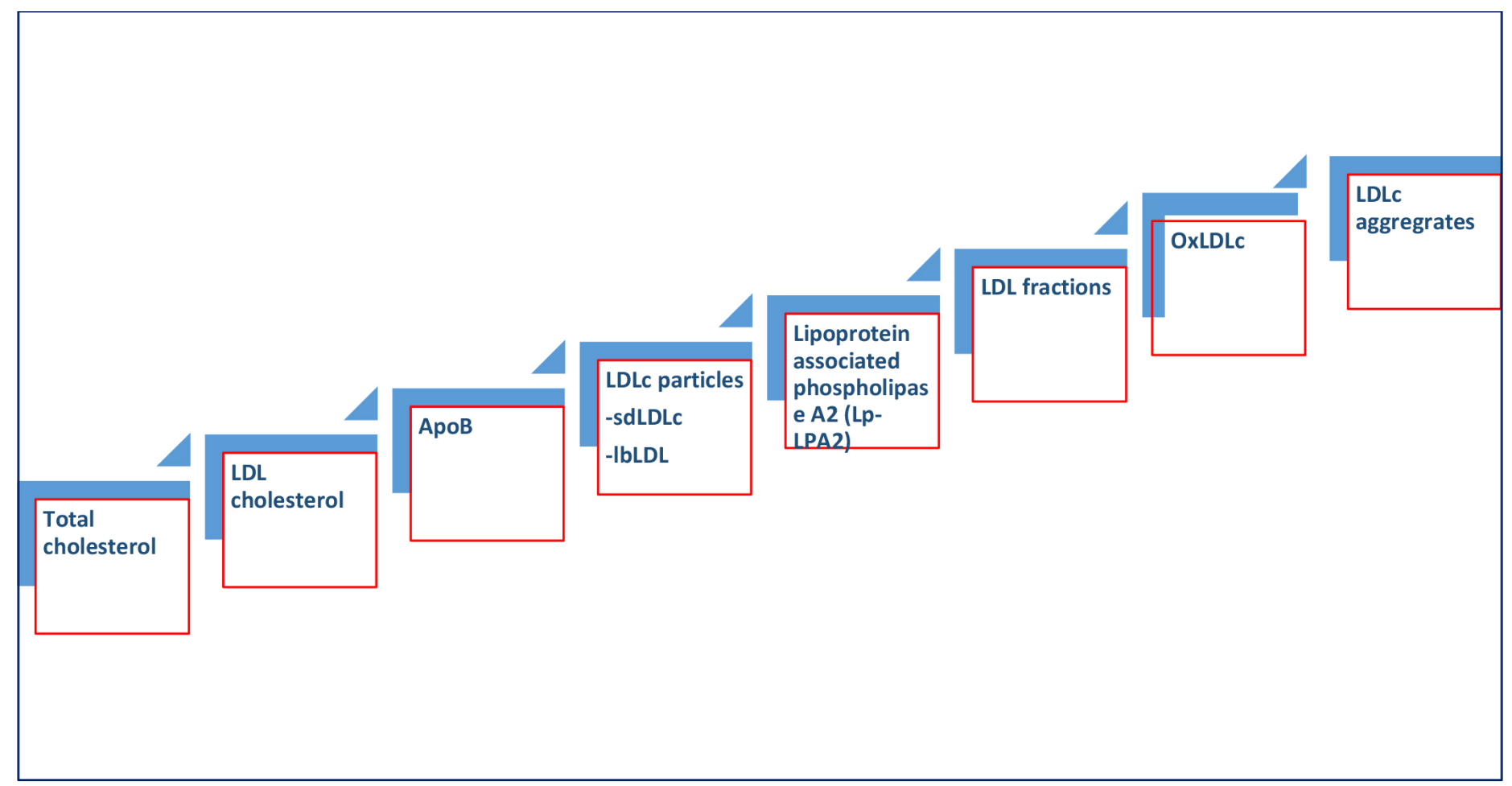

Figure 2. Evolution LDL biomarkers for predicting adverse ASCVD consequences

\section{References}

1. Oncley JL, Gurd FRM, Melin M (1950) Preparation and properties of serum and plasma proteins XXV. Composition and properties of human serum $\beta$-lipoprotein. J. Am. Chem. Soc. 68: 458-464.

2. Piepoli MF, Hoes AW, Agewall S, Albus C, Brotons C, Catapano AL, et al. (2016) ESC Scientific Document Group. 2016 European Guidelines on cardiovascular disease prevention in clinical practice: The Sixth Joint Task Force of the European Society of Cardiology and Other Societies on Cardio vascular Disease Prevention in Clinical Practice (constituted by representatives of 10 societies and by invited experts) Developed with the special contribution of the European Association for Cardiovascular Prevention \& Rehabilitation (EACPR). Eur Heart J. 37(29): 23152381. doi: 10.1093/eurheartj/ehw106. [Crossref]

3. Otvos JD, Mora S, Shalaurova I, Greenland P, Mackey RH, Goff DC Jr. (2011) Clinical implications of discordance between low-density lipoprotein cholesterol and particle number. J Clin Lipidol. 5(2): 105-13. doi: 10.1016/j.jacl.2011.02.001. [Crossref]

4. Srisawasdi P, Vanavanan S, Rochanawutanon M, Kruthkul K, Kotani K, Kroll MH (2015) Small-dense LDL/large-buoyant LDL ratio associates with the metabolic syndrome. Clin Biochem. 48(7-8): 495-502. doi: 10.1016/j. clinbiochem.2015.01.011. [Crossref]

5. Kulkarni KR (2006) Cholesterol profile measurement by vertical auto profile method. Clin Lab Med. 26(4): 787-802. [Crossref]

6. Miller WG, Myers GL, Sakurabayashi I, Bachmann LM, Caudill SP, Dziekonski A, et al. (2010) Seven direct methods for measuring HDL and LDL cholesterol compared with ultracentrifugation reference measurement procedures. Clin Chem. 56(6): 977-86. doi: 10.1373/clinchem.2009.142810. [Crossref]

7. Ramasamy I (2018) Update on the laboratory investigation of dyslipidemias. Clin Chim Acta. 479: 103-125. doi: 10.1016/j.cca.2018.01.015. [Crossref]

8. Silva IT, Almeida-Pititto Bd, Ferreira SR (2015) Reassessing lipid metabolism and its potentialities in the prediction of cardiovascular risk. Arch Endocrinol Metab. 59(2): 171-80. doi: 10.1590/2359-3997000000031. [Crossref]

9. Lawler PR, Akinkuolie AO, Chu AY, Shah SH, Kraus WE, Craig D, et al. (2017) Atherogenic Lipoprotein Determinants of Cardiovascular Disease and Residual Risk Among Individuals With Low Low-Density Lipoprotein Cholesterol. $J$ Am Heart Assoc. 6(7). pii: e005549. doi: 10.1161/JAHA.117.005549. [Crossref]

10. Diffenderfer MR, Schaefer EJ (2014) The composition and metabolism of large and small LDL. Curr Opin Lipidol. 25(3): 221-6. doi: 10.1097/ MOL.0000000000000067. [Crossref]

11. Gerber PA, Nikolic D, Rizzo M (2017) Small dense LDL: an update. Curr Opin Cardiol. 32(4): 454-459. doi: 10.1097/HCO.0000000000000410. [Crossref]
12. Williams K, Sniderman AD, Sattar N, D'Agostino R Jr, Wagenknecht LE, Haffner SM (2003) Comparison of the associations of apolipoprotein B and low-density lipoprotein cholesterol with other cardiovascular risk factors in the Insulin Resistance Atherosclerosis Study (IRAS). Circulation. 108(19): 2312-6. [Crossref]

13. Fernández-Friera L, Fuster V, López-Melgar B, Oliva B, García-Ruiz JM, Mendiguren J, et al. (2017) Normal LDL-Cholesterol Levels Are Associated With Subclinical Atherosclerosis in the Absence of Risk Factors. J Am Coll Cardiol. 70(24): 2979-2991. doi: 10.1016/j.jacc.2017.10.024. [Crossref]

14. Sniderman AD, Robinson JG (2018) ApoB in clinical care: Pro and Con. Atherosclerosis. pii: S0021-9150(18)31456-4. doi: $10.1016 / \mathrm{j}$. atherosclerosis.2018.11.001. [Crossref]

15. Lp-PLA (2) Studies Collaboration, Thompson A, Gao P, Orfei L, Watson S, Di Angelantonio E, Kaptoge S, et al. (2010) Lipoprotein-associated phospholipase $\mathrm{A}(2)$ and risk of coronary disease, stroke, and mortality: collaborative analysis of 32 prospective studies. Lancet. 375(9725): 1536-44. doi: 10.1016/S01406736(10)60319-4. [Crossref]

16. Anderson JL (2008) Lipoprotein-associated phospholipase A2: an independent predictor of coronary artery disease events in primary and secondary prevention. Am J Cardiol. 101(12A): 23F-33F. doi: 10.1016/j.amjcard.2008.04.015. [Crossref]

17. Ridker PM, MacFadyen JG, Wolfert RL, Koenig W (2012) Relationship of lipoprotein-associated phospholipase A2mass and activity with incident vascular events among primary prevention patients allocated to placebo or to statin therapy: an analysis from the JUPITER trial. Clin Chem 58: 877-86.

18. Heart Protection Study Collaborative Group. Lipoprotein-associated phospholipase A2 activity and mass in relation to vascular disease and nonvascular mortality. $J$ Intern Med 2010;268: 348-58. [Crossref]

19. McConnell JP, Jaffe AS (2008) Variability of lipoprotein-associated phospholipase A2 measurements. Clin Chem. 54(5): 932-3. doi: 10.1373/clinchem.2008.103358. [Crossref]

20. Gourgari E, Lodish M ,Shamburek R, Keil M, Wesley R, Walter M, et al. (2015) Lipoprotein Particles in Adolescents and Young Women With PCOS Provide Insights Into Their Cardiovascular Risk. J Clin Endocrinol Metab. 100(11): 42918. doi: 10.1210/jc.2015-2566. [Crossref]

21. Steffen BT, Guan W, Remaley AT, Paramsothy P, Heckbert SR, McClelland RL, et al. (2015) Use of lipoprotein particle measures for assessing coronary heart disease risk post-American Heart Association/American College of Cardiology guidelines: the Multi-Ethnic Study of Atherosclerosis. Arterioscler Thromb Vasc Biol. 35(2): 448-54. doi: 10.1161/ATVBAHA.114.304349. [Crossref]

22. Choi SH, Sviridov D, Miller YI (2017) Oxidized cholesteryl esters and inflammation. Biochim Biophys Acta Mol Cell Biol Lipids. 1862(4): 393-397. doi: 10.1016/j.bbalip.2016.06.020. [Crossref] 
Sikandar Hayat Khan (2019) LDL and beyond: New emerging LDL biomarkers in lipidology

23. Laufs U, Weingärtner O (2018) Pathological phenotypes of LDL particles. Eur Heart J. 39(27): 2574-2576. doi: 10.1093/eurheartj/ehy387.

24. Deevska GM, Sunkara M, Morris AJ, Nikolova-Karakashian MN (2012) Characterization of secretory sphingomyelinase activity, lipoprotein sphingolipid content and LDL aggregation in ldlr-/- mice fed on a high-fat diet. Biosci Rep. 32(5): 479-90. doi: 10.1042/BSR20120036. [Crossref]
25. Ruuth M, Nguyen SD, Vihervaara T, Hilvo M, Laajala TD, Kondadi PK, et al. (2018) Susceptibility of low-density lipoprotein particles to aggregate depends on particle lipidome, is modifiable, and associates with future cardiovascular deaths. Eur Heart J. 39(27): 2562-2573. doi: 10.1093/eurheartj/ehy319. [Crossref]

\section{Citation:}

Sikandar Hayat Khan (2019) LDL and beyond: New emerging LDL biomarkers in lipidology. J Clin Res Med Volume 2(2): 1-5. 